\title{
A NOTE ON PREPROJECTIVE PARTITIONS OVER HEREDITARY ARTIN ALGEBRAS
}

\author{
GORDANA TODOROV
}

\begin{abstract}
If $\Lambda$ is an artin algebra there is a partition of ind $\Lambda$, the category of indecomposable finitely generated $\Lambda$-modules, ind $\Lambda=\cup_{i>0} P_{i}$, called the prepro: jective partition. We show that $\underline{P}_{i}$ can be easily constructed for hereditary artin algebras, if $\underline{P}_{i-1}$ is known: $A$ is in ${ }^{P_{i}}$ if and only if $A$ is not in $\underline{P}_{i-1}$ and there is an irreducible map $B \rightarrow A$, where $B$ is in $\underline{P}_{i-1}$.
\end{abstract}

If $\Lambda$ is a hereditary artin algebra V. Dlab and C. M. Ringel called an indecomposable module $X$ preprojective if there exist an indecomposable projective $P$ and an integer $j$ such that $X \cong \operatorname{Tr} D^{j} P$, where $\operatorname{Tr}$ denotes the transpose and $D$ the ordinary duality. M. Auslander and S. Smalo gave a definition of preprojective modules for arbitrary artin alegbras, which in the hereditary case coincides with the previous one. They proved [2] that for any artin algebra there exist unique sets of isomorphism classes of indecomposable modules $\underline{\underline{P}}_{i}, i \in N \cup\{\infty\}$ such that:

(i) $\underline{P}_{0}$ consists of the indecomposable projective $\Lambda$-modules.

(ii) $\underline{P}_{n}$ is finite for each $n<\infty$ and is minimal with respect to the property that each indecomposable module not in $\cup_{i=0}^{n-1} \underline{P}_{i}$ is a quotient of a direct sum of modules from $\underline{P}_{n}$.

(iii) $\underline{P}_{=}$is the collection of indecomposable modules not in $\cup_{i<\infty} \underline{P}_{i}$. A module is called preprojective if it is in $\cup_{i<\infty} \stackrel{P}{=}_{i}$.

One of the basic tools in describing preprojective partitions is irreducible maps defined by $M$. Auslander and $\mathrm{I}$. Reiten in [1]. A nonzero map $f: M \rightarrow N$ is irreducible if $f$ is neither a splittable monomorphism nor a splittable epimorphism and, given any factorization of $f$,

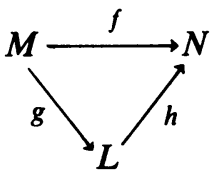

then either $\boldsymbol{g}$ is a splittable monomorphism or $\boldsymbol{h}$ is a splittable epimorphism.

The following result about preprojective modules is true for arbitrary artin algebras [3].

(a) $A \in \underline{P}_{0}$ if and only if $A$ is an indecomposable projective module.

Received by the editors April 14, 1981.

1980 Mathematics Subject Classification. Primary 16A64; Secondary 16A35.

(C)1982 American Mathematical Society 0002-9939/81/0000-1078/\$02.25 
(b) $A \in P_{1}$ if and only if $A \cong \operatorname{Tr} D B$ for some summand $B$ of the radical of $\Lambda$ as left module.

(c) If $A \in \underline{P}_{n}$, then there exist a $B$ in $\underline{P}_{j}$ for some $j<n$ and an irreducible morphism $B \stackrel{\text { n }}{\rightarrow} A$.

This result gives a complete description of the modules in $P_{0}$ and $P_{1}$, and a way to find $\underline{P}_{n}, n<\infty$, when all the $\underline{P}_{j}, j<n$ are given, which is described in the same paper. First look at the set $A_{n}$ of all indecomposable modules $A$ such that there exists an irreducible morphism $f: \vec{B} \rightarrow A$ for some $B$ in $\cup_{i<n} \stackrel{P}{=}_{i} \cdot \cup_{i<n} \underline{P}_{i}$ is finite so the set $A_{n}$ will also be finite [1]. Further it is possible to prove that ${ }_{n} n$ is a cover for ind $\Lambda-\cup_{i<n} \stackrel{P}{=}$ [2], and therefore it contains a cover with a minimal number of elements which then is the unique minimal cover $\underline{P}_{n}$ of ind $\Lambda-\cup_{i<n} P_{i}$. To determine the minimal cover one can start with any $A$ in $\stackrel{P}{=}_{n}$ and see if $\bar{A}_{n}-\{\overline{\bar{A}}\}$ is a cover for $A_{n} . A$ is then in the minimal cover $P_{n}$ if and only if $A_{n}-\{A\}$ is not a cover for $A_{n}$. Hence, by going through all modules in $\underline{=}_{n}$ in an arbitrary order one can find the members of $\underline{P}_{n}$.

If $\Lambda$ is a hereditary artin algebra, instead of condition (c), we give necessary and sufficient conditions for a module $A$ to be in $P_{n}: A$ is in $P_{n}$ if and only if it not in $\stackrel{P}{=n-1}_{n}$ and there is an irreducible map $B \rightarrow \overline{\bar{A}}^{n}$, where ${ }_{B}{ }^{n}$ is in $P_{n-1}$. This way the $\overline{\bar{c}}^{n-1}$ struction described before becomes much simpler: it is enough to consider the set $A_{n}$ and exclude all modules that are in $P_{n-1}$ in order to obtain $P_{n}$, which means just $\overline{\bar{b}}^{n}$ looking at the modules and without $\overline{\bar{c}}^{n-1}$ insidering maps and possible covers we can determine which modules are in $\underline{P}_{n}$.

Dually, one can define preinjective partitions and the dual statements hold. D. Zacharia applied these results to obtain some interesting results about preprojective and preinjective partitions for hereditary and stably equivalent to hereditary artin algebras [4].

Proposition 1. Let $\Lambda$ be a hereditary artin algebra. Then $Y$ is in $\underline{P}_{n}$ if and only if both of the following conditions hold: and

(a) For every irreducible map $f: X \rightarrow Y$ with $X$ indecomposable, $X$ is in $\underline{P}_{n-1} \cup \underline{\underline{P}}_{n}$,

(b) there is an indecomposable $X_{n-1}$ in $\underline{=}_{n-1}$ and an irreducible map $f: X_{n-1} \rightarrow Y$.

Proof. (a) The proof will be by induction on $n$. If $n=0$, it is clear since $\Lambda$ is hereditary. Suppose it is true for $j \leqslant n$. Let $A_{n+1}$ be in $P_{n+1}$ and suppose $X \rightarrow A_{n+1}$ is irreducible, and $X$ is indecomposable. Suppose $X$ is in $\underline{P}_{t}$ for some $t \leqslant n-1$. Consider an almost split sequence

$$
0 \rightarrow C \rightarrow X \amalg B \rightarrow A_{n+1} \rightarrow 0 .
$$

Let $B_{n-1}^{\prime}$ be a summand of $B$ containing all summands of $B$ which are in $\cup_{k \leqslant n-1} \underline{P}_{k}$, and $B^{\prime}$ is determined by $B=B_{n-1}^{\prime} \amalg B^{\prime}$. Then $B^{\prime}$ has no summands from $\cup_{k<n-1} \underline{\bar{P}}_{k}^{k}$. So (1) looks like:

$$
0 \rightarrow C \rightarrow X \amalg B_{n-1}^{\prime} \amalg B^{\prime} \rightarrow A_{n+1} \rightarrow 0 .
$$


Since $\underline{P}_{n}$ is a generating set for $\Lambda-\cup_{k<n} \underline{P}_{n}$, there exists an epimorphism $f$ : $\amalg Y_{n} \rightarrow \stackrel{P}{A}_{n+1}^{n}$ with all $Y_{n}$ in $\underline{\underline{P}}_{n}$.

ClaIM. If $X$ is in $\cup_{k<n} \stackrel{\bar{P}}{=}_{k}^{n}$, then $\operatorname{Hom}_{\Lambda}\left(Y_{n}, X\right)=0$.

Proof of THE Claim. Suppose $X$ is in $P_{n-1}$. Call it $X_{n-1}$. Let $f$ be a nonzero map $Y_{n} \rightarrow X_{n-1}$. We may assume that $X_{n-1} \overline{\text { is }}^{n-1}$ not projective. Consider an almost split sequence $0 \rightarrow K \rightarrow \amalg Z_{k} \rightarrow X_{n-1} \rightarrow 0$. By induction hypothesis all $Z_{k}$ 's are in $P_{n-2} \cup P_{n-1}$. So $f$ factors through some $Z_{k}$ which is either in $\underline{P}_{n-2}$ or $\underline{P}_{n-1}$. Clearly, if $Z_{k}$ is $\stackrel{\text { in }}{n}_{n-1}^{n-1}$, then irreducible map $Z_{k} \rightarrow X_{n-1}$ is a monomorphism. Applying the same argument either we reach some module in $\cup_{k<n} \stackrel{P}{k}_{k}$ which is isomorphic to $Y_{n}$, which is impossible since $Y_{n}$ is in $\underline{P}_{n}$, or we have an infinite chain of irreducible monomorphisms in the same $\underline{P}_{k}$, which is again impossible, or we have a map from $Y_{n}$ to a projective, which is impossible since $\Lambda$ is hereditary. This finishes proof of the claim.

Therefore $\operatorname{Hom}_{\Lambda}\left(Y_{n}, X\right)=0$ and $\operatorname{Hom}_{\Lambda}\left(Y_{n}, B_{n-1}^{\prime}\right)=0$. So $f$ factors through $B^{\prime}$. Thus $B^{\prime} \rightarrow A_{n+1}$ is an epimorphism, which implies that $C \rightarrow X$ is an epimorphism. Since $X$ is in $\underline{P}_{t}$, the module $C$ cannot be in $P_{t}$, otherwise $\underline{P}_{t}$ would not be a minimal generating set, and by the induction hypothesis $C$ cannot ${ }^{t}$ in $\cup_{j<t-1} P_{j}$. Thus $C$ is in $P_{t-1}$. Then we will denote $C$ by $C_{t-1}$. (Similarly we will write $X=X_{t}$ since $X$ is in $\left.\underline{P}_{t}.\right)$ So the sequence (1) is

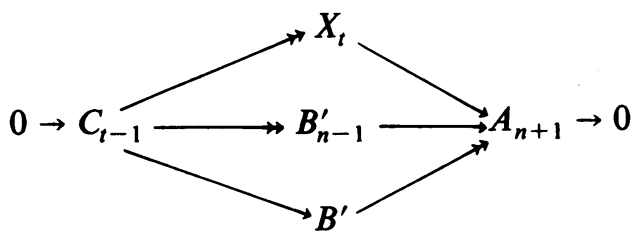

Not all summands of $B^{\prime}$ can be in $\cup_{k>n+1} \underline{P}_{k}$, otherwise $A_{n+1}$ would not be in the minimal generating set $P_{n+1}$. Therefore there exists a summand of $B^{\prime}$ which is in $P_{n}$. Call it $B_{n}$. But then from the above sequence we have an irreducible map $C_{t-1} \rightarrow \bar{B}_{n}^{n}$, which is a contradiction to the induction hypothesis, since $t-1<n-1$.

(*) Therefore if there is an irreducible map $X_{t} \rightarrow A_{n+1}$, where $X_{t}$ is in $\underline{P}_{t}$, then $t \geqslant n$.

Now suppose there is an irreducible map $X_{t} \rightarrow A_{n+1}$ with $X_{t}$ in $\underline{P}_{t}$ and $t>n+1$. Consider an almost split sequence

$$
0 \rightarrow C \rightarrow X_{t} \amalg B \rightarrow A_{n+1} \rightarrow 0 .
$$

By condition (c) which was stated for arbitrary artin algebras there exists a summand of $B$ which is in $P_{k}$ with $k \leqslant n$. But by (*) $k \geqslant n$, so $k=n$. So the above sequence looks like $0 \rightarrow C \stackrel{{ }^{k}}{\rightarrow} X_{t} \amalg B^{\prime} \amalg B_{n} \rightarrow A_{n+1} \rightarrow 0$ where $B \cong B^{\prime} \amalg B_{n}$ and $B_{n}$ is in $\underline{P}_{n}$. Since there is an irreducible map $C \rightarrow B_{n}$, by the induction hypothesis, $C$ is in $\underline{P}_{s}$ 
with $n-1 \leqslant s \leqslant n$. We denote $C$ by $C_{s}$. Consider now an almost split sequence for $X_{t}$ :

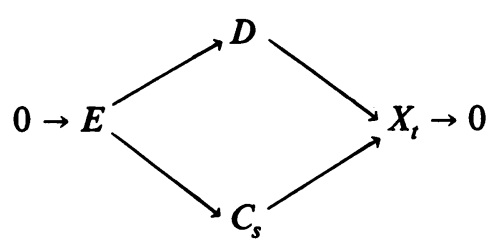

Since $t \geqslant n+2$, there exists an epimorphism $f: Y \rightarrow X_{t}$ with all summands of $Y$ in $\underline{P}_{n+1}$. Let $D_{n}^{1}$ be a summand of $D$ containing all summands which are in $\cup_{k<n} \underline{P}_{k}$. Let $D=D^{1} \amalg D_{n}^{1}$. Then $\operatorname{Hom}_{\Lambda}\left(Y, D_{n}^{1}\right)=0$, and also $\operatorname{Hom}_{\Lambda}\left(Y, C_{s}\right)=0$. So $f$ factors through $D^{1}$ and $D^{1} \rightarrow X_{t}$ is an epimorphism. Thus the previous sequence looks like

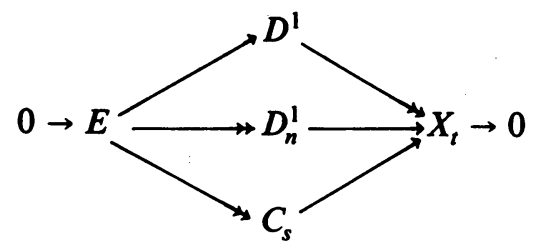

So there exists a summand $D_{j_{1}}$ of $D^{1}$, with $D_{j_{1}}$ in $P_{j_{1}}$ with $j_{1}<t$ since $X_{t}$ is in $P_{t}$ which is a minimal generating set. Also $n+1 \leqslant j_{1}$ since $D_{j_{1}}$ is a summand of ${ }^{t} D^{1}$. So $n+1 \leqslant j_{1}<t$. Since the map $E \rightarrow C_{s}$ is an epimorphism, by the induction hypothesis $E$ is in $P_{s-1}$. Denote $E=E_{s-1}$. Then there is an irreducible map $E_{s-1} \rightarrow D_{j_{1}}$. If $j_{1}=n+1$, then by $(*) s-1 \geqslant n$, which is a contradiction since $s \leqslant n$. Therefore $n+1<j_{1}<t$.

Consider now an almost split sequence:

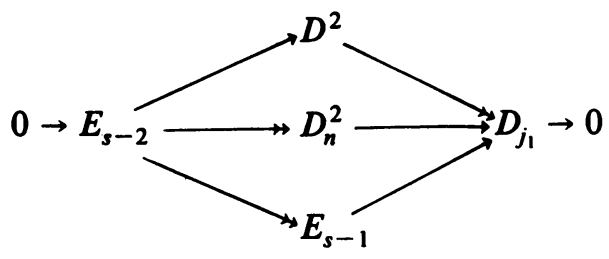

where $D^{2}$ has no summand from $\cup_{j<n} P_{j}$, and $D_{n}^{2}$ contains all such summands except for $E_{s-1}$. Then by the argument as before, $D^{2} \rightarrow D_{j_{1}}$ is an epimorphism, $E_{s-2} \rightarrow E_{s-1}$ is an epimorphism, and $E_{s-2}$ is in $P_{s-2}$. Using the same argument that we used to get $D_{j_{1}}$, we can show that there is a summand $D_{j_{2}}$ of $D^{2}$ with $D_{j_{2}}$ in $P_{j_{2}}$ and $n+1<j_{2}<j_{1}$. This way we obtain a chain of decreasing positive integers, which are all larger than $n+1$, giving a contradiction.

(b) This now follows from part (a) and the condition (c) stated for arbitrary artin algebras. 
COROLlaRY 2. Let $\Lambda$ be a hereditary artin algebra and let $A_{n}=\{X$ in ind $\Lambda \mid$ there exists an irreducible map $A_{n-1} \rightarrow X$ with $A_{n-1}$ in $\left.\underline{P}_{n-1}\right\}$. Then $\underline{\underline{P}}_{n}=\underline{A}_{n}-\underline{P}_{n-1}$, for $n \geqslant 1$.

Proof. Suppose $Y$ is in $\underline{P}_{n}$. Then if $X \rightarrow Y$ is irreducible, $X$ is in $\underline{P}_{n} \cup \vec{P}_{n-1}$ by Proposition 1(a), and by (b) there exist $X_{0}$ in $P_{n-1}$ and an irreducible map $\overline{\bar{X}}_{0}^{n} \rightarrow Y$. So $Y$ is in $A_{n}$, and since $P_{i}$ 's are disjoint, $Y$ is in $\underline{A}_{n}-P_{n-1}$. Now suppose $Y$ is in $A_{n}-\underline{P}_{n-1}$. Then there exists an irreducible map $A_{n-1} \rightarrow Y$ with $A_{n-1}$ in $\underline{P}_{n-1}$. Then by Proposition 1, $Y$ is in $\underline{P}_{n-1} \cup \underline{P}_{n}$, and therefore $Y$ is in $\underline{P}_{n}$.

COROllary 3. Suppose $X$ is in $\boldsymbol{P}_{n}$. Then there exists a chain of irreducible maps $X_{0} \rightarrow X_{1} \rightarrow \cdots \rightarrow X_{j}=X$ with $X_{0} \overline{\bar{p}}^{n^{*}}$ rojective, and the shortest such chain is of length $n$.

Proof. If $X$ is in $P_{n}$, then there exist a module $X_{n-1}$ in $P_{n-1}$ and an irreducible map $X_{n-1} \rightarrow X$. App广ying the same argument, we obtain a chain $X_{0} \rightarrow X_{1} \rightarrow \cdots \rightarrow$ $X_{n-1} \rightarrow X$, with $X_{k}$ in $\underline{P}_{k}$ for all $0 \leqslant k \leqslant n-1$. Let $X_{0}^{\prime} \rightarrow X_{1}^{\prime} \rightarrow \cdots \rightarrow X_{j-1}^{\prime} \rightarrow X_{j}^{\prime}=$ $X$ be the shortest chain of irreducible maps with $X_{0}^{\prime}$ in $\underline{P}_{0}$. Then by Proposition 1 if $X_{s}^{\prime}$ is in $\underline{P}_{s}$, the module $X_{s+1}^{\prime}$ is in $\underline{P}_{s} \cup \vec{P}_{s+1}$. So we have $X_{1}^{\prime}$ is in $P_{0} \cup P_{1}$, and $X_{k}^{\prime}$ is in $\cup_{i<k} \stackrel{P}{P}_{i}$ for all $k \leqslant j$. So $X_{j}^{\prime}$ is in $\bar{U}_{i<j}^{s}{ }_{i}$, and since $X_{j}^{\prime}=X$ which is in $\underline{P}_{n}$, it follows that $n \leqslant j$. But $j$ was chosen to be minimal, so $n=j$.

To illustrate the results, we give an example of a hereditary artin algebra, the first few classes of the partition $\cup \boldsymbol{P}_{i}$, and the irreducible maps between the modules in these classes: let $k$ be a field $\bar{\Lambda}$ the subring of $8 \times 8$ lower triangular matrix ring, given by

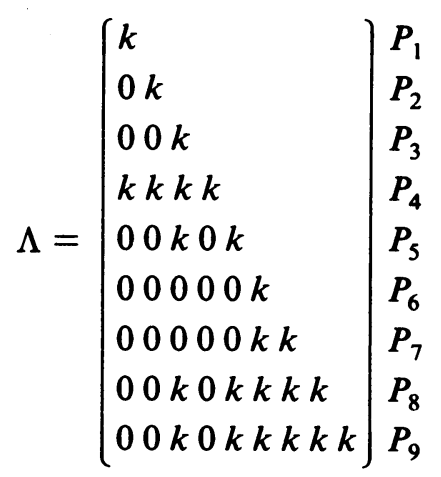

where the $P_{i}$ 's are indecomposable right projective $\Lambda$-modules. This ring corresponds to the ring given by the diagram

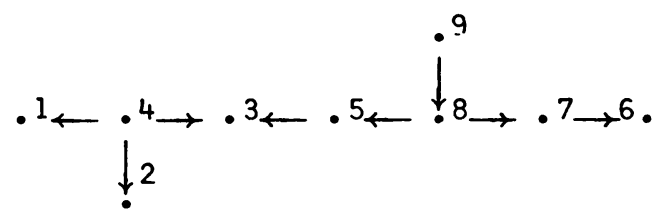


This ring is of infinite representation type, and a part of the Auslander-Reiten graph looks like

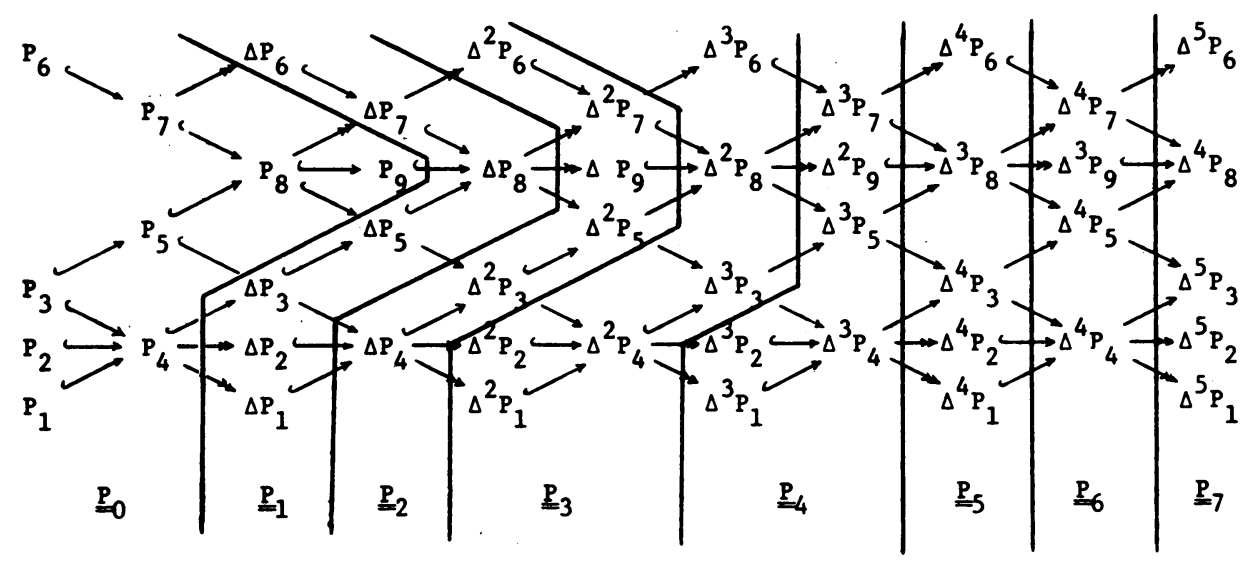

where $\Delta$ denotes $\operatorname{Tr} D$.

\section{REFERENCES}

1. M. Auslander and I. Reiten, Representation theory of artin algebras. IV. Invariants given by almost split sequences, Comm. Algebra 5 (1977), 441-518.

2. M. Auslander and S. Smalo, Preprojective modules over artin algebras, J. Algebra 66 (1980), 61-122.

3. - Preprojective modules: an introduction and some applications, Lecture Notes in Math., Vol. 831, Springer-Verlag, Berlin and New York, 1979, pp. 48-73.

4. D. Zacharia, The preprojective partitions for hereditary artin algebras, Ph.D. Thesis, Brandeis University, 1981.

Department OF Mathematics, NortheAstern University, Boston, MASSAChuSETtS 02115 